\title{
TGFbeta Receptor Ectodomain-IgG Fc Fusion Protein AVID200
}

National Cancer Institute

\section{Source}

National Cancer Institute. TGFbeta Receptor Ectodomain-Ig G FC Fusion Protein AVID200.

NCI Thesaurus. Code C159817.

A fusion protein composed of the ectodomain of the transforming growth factor (TGF) beta (T GF-beta; T GFb) receptor fused to the human immunog lobulin G (IgG) Fc domain, with potential antineoplastic, immunomodulating and anti-fibrotic activities. Upon administration of the TGFb receptor ectodomain-IgG Fc fusion protein AVID200, the fusion protein specifically and selectively targets, binds to and neutralizes the T GF ligands T GF-beta isoform 1 (T GFb1) and 3 (T GFb3). This prevents T GF ligands from binding to TGF receptors and prevents T GFb-mediated signaling. This abrog ates T GFb1/3mediated immunosuppression, enhances anti-tumor immunity in the tumor microenvironment (TME) and promotes a cytotoxic T-lymphocyte (CTL)-mediated immune response ag ainst tumor cells leading to tumor cell death. This may lead to a reduction in T GFb-dependent proliferation of cancer cells. By preventing TGFb1/3mediated signaling, AVID200 also prevents bone marrow fibrosis and promotes the proliferation of normal hematopoietic progenitors. TGFb, overproduced in myelodysplastic syndrome (MDS) and in many other types of cancer, plays a key role in immunosuppression in the TME, enhances tumor cell proliferation, and promotes cancer progression. The TGFb1 and TGFb3 isoforms are negative regulators of hematopoiesis that play key roles in the pathogenesis and progression of fibrotic diseases. By selectively targeting only specific isoforms of T GFb with minimal activity ag ainst T GFb2, AVID200 minimizes the potential for cardiotoxicity and promotion of metastasis. The TGFb2 isoform promotes hematopoiesis and plays a key role in normal cardiac function while the inhibition of the TGFb2 isoform promotes metastasis. 